\title{
Potential usefulness of CTC detection in follow up of prostate cancer patients. A preliminary report obtained by using Adnagene platform
}

\author{
Giuseppe Albino ${ }^{1,2}$, Francesca Vendittelli ${ }^{1}$, Carmela Paolillo ${ }^{1}$, Cecilia Zuppi ${ }^{1}$, \\ Ettore Capoluongo ${ }^{1}$ \\ ${ }^{1}$ Laboratory of Molecular Diagnostics, Institute of Biochemistry and Clinical Biochemistry, Catholic \\ University, Rome, Italy; \\ ${ }^{2}$ Unit of Urology, Ospedale di Andria, ASL BAT, Italy.
}

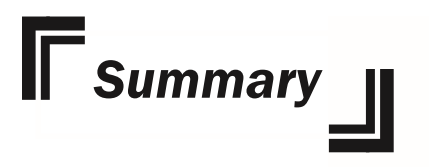

Objective: Prostate cancer (PCa) represents one of the most important medical problems for males, being the second major cause of cancer death. Routinely, PCa patients are followed up with both periodic evaluation of serum PSA levels and imaging. Recently, alternative laboratory methods were proposed for PCa patients' monitoring, with contrasting results.

Aim of the present study was to evaluate the usefulness of a new commercially CE-IVD kit for detection of prostate circulating tumour cells. Our intention was to verify the Adnagene platform usefulness to identify patients with disease progression, whatever treatment ongoing, in order to modify the therapeutic process even before treatment failure is evident with imaging methods.

Materials and Methods: Twenty-one patients were enrolled and subdivided into three groups: $n=10$ high risk tumor $P C a$ patients; $n=6$ low risk $P C a$ patients; $n=5$ sbjects without any signs of PCa. AdnaTest Prostate Cancer kit was used for enrichment and molecular characterization of prostate circulating tumour cells.

Results: Healthy subjects (with BPH) and patients without metastases resulted as negative, while 3 out of 10 high risk PCa patients were positive at least for one molecular marker like PSA, while only two showed positivity for PSMA mRNA. Our results indicate that the test specificity is 100\% and the sensitivity is 100\%; of course the sample is too small to give it statistical validity. In detail we verified that only the "not responder" patients resulted positive for AdnaTest.

Conclusions: The present preliminary report provides evidence that isolation and detection of circulating tumour cells (CTCs) is feasible and it may be useful in the follow-up of patients with advanced prostate cancer. If the results of this preliminary study would be confirmed by a large prospective cohort study, it could be demonstrated that this test is a rapid diagnostic method, based on the analysis of a blood sample and useful to the clinician to decide when to change therapy for patients resistant to castration or able to confirm that, at that time, the therapy is effective.

KEY WORDS: Prostate cancer; Circulating prostate tumor cells; AdnaTest.

\section{INTRODUCTION}

Prostate cancer ( $\mathrm{PCa}$ ) represents the second major cause of cancer death in men (1). Radical prostatectomy (RP) and radiotherapy (RT) are well-established primary therapeutic options for management of localized PCa, although cancer recurrence still remains a significant concern for patients. In fact, about $27-53 \%$ of patients undergoing RT or RP will develop local or at distance metastases within 10 years from initial treatment and 16$35 \%$ of PCa patients will receive second-line treatment within 5 years of initial therapy $(2,3)$. Routinely, PCa patients are followed up with periodic evaluation of serum prostate-specific antigen (PSA) levels and imaging 
(TC, RMN, Bone Scintigraphy, 18-F Choline PET) (4), although undetectable serum PSA does not necessarily indicate the absence of any potential metastatic risk.

In this way, the release of tumor cells into bloodstream could represent a peculiar sign of cancer aggressiveness both before and after therapy $(5,6)$ : therefore, circulating tumour cells (CTCs) detection could provide precious informations to the clinician regarding disease status. In this context, due to the lack of additional markers able to early detect PCa relapse, use of reliable test for prostate CTCs detection could provide novel opportunities for alternative therapeutic approaches $(7,8)$.

Recent studies employed RT-PCR methods for detection of prostate-specific mRNAs in whole blood without any pre-selection of CTCs $(9,10)$. Prostate-specific mRNAs are considered as surrogate markers of CTC presence, although with conflicting results $(11,12)$. However, recent studies on individuals with prostate cancer at advanced stages, performed using the CellSearch platform (13-15), found that high CTC counts correlated with poor prognosis (13-18). CTC detection rates are really variable among studies, ranging from $54 \%$ to $62 \%$, mainly depending on the characteristics of the patients studied (19-20). Some methods for CTC characterization in PCa patients are in developing or under clinical laboratory evaluation $(20,21)$.

A recent CE-IVD method, namely AdnaTest Prostate Cancer Select plus AdnaTest Prostate Cancer Detect (AdnaGen AG, Langenhagen, Germany), has been commercialized for laboratory CTCs detection through multiple amplification for PCa-associated transcripts (reviewed as possible useful markers for monitoring of PCa patients) (22-24).

In the present work, we evaluated AdnaGene method for discriminating PCa patients at risk for metastasis from those with remission or stable disease, in order to establish if this method may be predictive or not of disease recurrence, with any ongoing therapy.

We describe preliminary data that might suggest the use of this method for management of PCa patients and support the clinical utility of the assay here described.

\section{Materials AND MEthods}

\section{Patients selection and blood sampling (more details reported in supplemental files)}

We selected 21 patients who were subdivided into three groups (Table 1), according to D'Amico (25) classification: $\mathrm{n}=10$ PCa patients belonging to Group I (conventionally defined as prognostic grouping stage IV), because of the high risk tumor class (Gleason Score (GS) $=8-10$ or TNM $>$ T2b NO MO or PSA > 20); n = 6 PCa patients included into Group II (conventionally defined as prognostic grouping stage I), who were defined as at low risk tumor class $(\mathrm{GS}=2-6$ and $\mathrm{TNM}=\mathrm{T} 1-\mathrm{T} 2 \mathrm{a}$ and PSA $<10) ; \mathrm{n}=5$ patients with benign prostate hypertrophy, without any clinical and biochemical signs of PCa, used as negative controls (defined as Group III; (PSA $<2$ and negative Digital Rectal Exploration or TRUS). Group III cases were well known patients, with PSA $<2$ for at least 5 years, taken from the database of our "stone center". Group II and
Group III were assayed as negative control groups. PCa patients belonging to Intermediate risk class ( $G S=7$ and $\mathrm{TNM}=2 \mathrm{~b}-\mathrm{T} 2 \mathrm{c}$ ) were excluded. The intermediate-risk patients were excluded due to their intermediate characteristics, concerning the risk of progression and recurrence of the disease. The design of the study aims to verify, without any doubt, that patients with localized disease or without prostatic cancer may never have circulating tumor cells detectable by this method (group II and group III). For logical reasoning, the only patients who might have CTCs are those belonging at high risk group (group I). In the design of the "case-control" study, the group to be studied is the "group I". Groups II and III are control groups. We felt it was a waste of resources the introduction of a further control group consisted of intermediate risk patients. Overall PCa patients (Groups I and II) were treated with single or combined therapy: radical prostatectomy, external beam radiotherapy, total androgen blockade; two patients of Group I started chemotherapy, because of their castration resistance. In order to reduce the biases due to the selection during the enrolment, we choose consecutively only patients who received a diagnosis of $\mathrm{PCa}$ within previous $36 \pm 4$ months, while the period of observation was 12 month from first blood sample collection for testing AdnaTest Prostate Cancer kit. In fact, for each patient enrolled we collected both $7 \mathrm{ml}$ of whole blood using specific preservative tubes for CTCs capture (AdnaGen, Langenhagen, Germany) and $3.5 \mathrm{ml}$ of vacutainer tubes for PSA measurement at diagnosis (PSA time $=0$ ). Additional PSA assays were done during the follow up period study (2 per year). Therapeutic information and clinical characteristics of patients were collected by a trained Urologist (Table I). All subjects enrolled in this preliminary investigation gave their informed consent. All procedures were made following the Helsinki criteria for research studies.

\section{AdnaTest Prostate Cancer select/detect}

Blood ( $5 \mathrm{ml}$ ) samples were taken using AdnaCollect blood collection tubes (AdnaGen, Langenhagen, Germany) and immediately processed not later than 24 hours after blood withdrawal, since it has been reported the possible lack of stability of cancer cell transcripts (26). The immunomagnetic enrichment of CTCs was performed by using AdnaTest Prostate Cancer Select and total mRNA/bead mixture was retro-transcribed by Sensiscript Reverse Transcriptase (Qiagen, Valencia, CA, USA) according to the manufacturer's instructions. Analysis of tumor-associated mRNAs, a multiplex PCR was carried out using AdnaTest Prostate Cancer Detect according to the manufacturer's instructions. The primer mixture consisted of amplified three tumour markers (PSMA, PSA, EGFR) and one housekeeping gene (Actin). Evaluations of cDNA run were carried out using Experion 1K analysis DNA Chip (Bio-Rad Hercules, $C A$ ) and $4 \%$ agarose gel.

\section{RESULTS}

For the correct interpretation of AdnaGene test results, Actin amplicons must be present in all patient's runs (internal PCR control): actin signal represents, in fact, the positive control for cell separation, reverse transcrip- 
Table 1.

Therapeutic informations and pathological characteristics of PCa and control patients, following the TNM classification (UICC, 2009).

\begin{tabular}{|c|c|c|c|c|c|c|c|}
\hline Patients & PSA t0 & $\begin{array}{c}\text { Prognostic } \\
\text { Grouping (stage) }\end{array}$ & GS & TNM & Therapy & Current PSA & ADNA MARKERS \\
\hline \multicolumn{8}{|l|}{ Group Ia } \\
\hline 1 & 332 & IV & $5+5$ & $\mathrm{~N}+\mathrm{M}+$ & enantone + casodex & 1,68 & NEG \\
\hline 2 & 12 & IV & $5+4$ & $\mathrm{~N}+\mathrm{M}+$ & eligard + casodex + RT + CHT & 0,98 & NEG/PSA* \\
\hline 3 & 136 & IV & $4+4$ & $\mathrm{~T} 4 \mathrm{~N}+\mathrm{M} 0$ & casodex $50+\mathrm{RT}$ & 0,49 & NEG \\
\hline 4 & 15 & IV & $3+5$ & $\mathrm{~N}+$ & gonapeptyl + casodex RT & 52 & PSA/PSMA \\
\hline 5 & 5,73 & IV & $4+4$ & pT4 & $\mathrm{RT}$ & 0,01 & NEG \\
\hline 6 & 7,05 & IV & $5+3$ & pT3b M+ & eligard + RT & 15 & PSA \\
\hline 7 & 128 & IV & $4+5$ & $\mathrm{~N}+\mathrm{M}+$ & eligard + casodex + taxotere & 68,5 & PSA/PSMA \\
\hline 12 & 9.8 & IV & $4+4$ & $M+$ & casodex + enantone + zometa & 9,29 & NEG \\
\hline 13 & 12 & IV & $4+4$ & $\mathrm{~T} 4$ & RT - Casodex suspension & 0,206 & NEG \\
\hline 14 & 8.9 & IV & $3+5$ & pT3b N+ & RRP R1 (margins +) & 0,03 & NEG \\
\hline \multicolumn{8}{|l|}{ Group II } \\
\hline 8 & 6,95 & I & $3+3$ & NO MO & RRP & 0,04 & $\mathrm{NEG}$ \\
\hline 9 & 9,24 & I & $3+2$ & NO MO & eligard & 0,04 & NEG \\
\hline 10 & 5,64 & i & $3+3$ & NO MO & decapeptyl + casodex + RT & 0,00 & NEG \\
\hline 11 & 9,83 & I & $3+3$ & NO MO & RT & 4,01 & NEG \\
\hline 15 & 4.32 & I & $3+3$ & NO MO & RRP & 4,32 & NEG \\
\hline 21 & 7.13 & I & $3+3$ & NO MO & casodex & 0,04 & NEG \\
\hline \multicolumn{8}{|l|}{ Group III } \\
\hline 16 & & & $\mathrm{BPH}$ & - & - & 0,174 & NEG \\
\hline 17 & & & BPH & - & - & 1,52 & NEG \\
\hline 18 & & & $\mathrm{BPH}$ & - & - & 0,267 & NEG \\
\hline 19 & & & $\mathrm{BPH}$ & - & - & 0,94 & NEG \\
\hline 20 & & & $\mathrm{BPH}$ & - & - & 1,94 & NEG \\
\hline
\end{tabular}

TNM = Cancer Staging System; GS = Gleason score (all GS are "biopsy GS" because they were not submitted to RRP; only pts 8-14-15 have "RP-GS");

ADNA MARKERS: PSA, PSMA, EGFR; BPH = benign prostate hypertrophy. All 15 healthy normal controls resulted as negative for the three ADNA markers.

a) Group I patients belong to stage IV of PCa disease, as reported in TNM classification of malignant tumors. UICC International Union Against Cancer. $7^{\text {th }}$ edn, 2009 (ref. 39).

b) As reported in the text, Group II patients includes low-risk progression PCa individuals, as indicated in the reference (25).

*Patient n. 2 was firstly negative at the AdnaGene test, but five month later (following the disease progression) resulted as positive.

tion and multiplex PCR. AdnaTest was considered positive for CTC presence if a PCR fragment of at least one tumor-associated transcript was clearly detectable and visible (peak concentration $\geq 10 \mathrm{ng} / \mu \mathrm{l}$ was fixed as the cut-off). Peaks outside the above described criteria were defined as inconclusive. All assays run on our samples passed the quality control criteria.

Results are briefly summarized in Table 1. As expected, all healthy individuals, control group III BPH patients, as well as those belonging to group II (who presented with disease properly controlled by therapy) resulted as negative for AdnaTest. Contrastingly, among patients belonging to Group I (staging class IV), AdnaTest identified as positive only those who were no more responsive to first- or second-line therapy, while as negative those responsive to first- or second-line chemotherapy, although being escaped by hormone therapy. Three out of ten PCa Group I patients resulted as positive for at least one tumor-associated marker like PSA, while only two showed positivity also for PSMA mRNA. It is important to underline that the Group I patient (listed as number 2 in Table 1), who was negative for AdnaTest during the first-line of chemotherapy, became positive for PSA marker five months later (as shown in the comparison between Figures 1A and1B). Surprisingly, EGFR mRNA resulted always negative in our patients: in order to establish if the negative results were dependent on limits of Adna-kit, we spiked mRNA extracted from prostate cancer slices positive at immunehistochemical analysis, and we found a perfect amplification of EGFR target (data not shown). Our results indicate that specificity of test is $100 \%$ and sensitivity was $100 \%$ (Table 2). In detail, individuals with BPH (group III) and those defined as "Stage I" (Group II) resulted as negative, while all patients belonging to Group I (defined as Stage IV) and responsive to therapy did not show any positivity for AdnaTest markers, while all "not responder" patients resulted positive for AdnaTest. The number of patients is too small to apply the tests of analysis of variance. Furthermore, Figure $1 \mathrm{~A}$ is representative of a group of patients' amplicons analyzed with Experion instrumentation. Clinical characteristics and main results of AdnaTest are synthetically reported below for each patient.

Details of clinical features of 15 PCa patients studied

Patient n. 1: showed a PSA relapse perhaps only for the infiltration of the bladder neck, but the bone and lymph node metastases were not metabolically active at scintigraphy examination: this test did not indicate the need of radiotherapy on the primitive mass;

Patient n. 2: was resistant to hormonal treatment. He initially responded to the first level chemotherapy, but subsequently his clinical conditions worsened. The AdnaTest 
Table 2.

Patient stratification based on response to single or combined therapies.

\begin{tabular}{|c|c|c|c|c|c|c|c|}
\hline Group I & Patients & PSA to & GS & TNM & Therapy & Current PSA & ADNA MARKERS \\
\hline \multicolumn{8}{|c|}{ Responders } \\
\hline & 1 & 332 & $5+5$ & $\mathrm{~N}+\mathrm{M}+$ & enantone + casodex & 1,68 & NEG \\
\hline & $2 *$ & 12 & $5+4$ & $\mathrm{~N}+\mathrm{M}+$ & eligard + casodex + RT + CHT & 0,98 & NEG \\
\hline & 3 & 136 & $4+4$ & $\mathrm{~T} 4 \mathrm{~N}+\mathrm{MO}$ & casodex $50+\mathrm{RT}$ & 0,49 & NEG \\
\hline & 5 & 5,73 & $4+4$ & pT4 & RT & 0,01 & NEG \\
\hline & 12 & 9.8 & $4+4$ & $\mathrm{M}+$ & casodex + enantone + zometa & 9,29 & NEG \\
\hline & 13 & 12 & $4+4$ & ТЗа & RT - Casodex suspension & 0,206 & NEG \\
\hline & 14 & 8.9 & $3+5$ & pT3b N+ & RRP R1 (margins +) & 0,03 & NEG \\
\hline \multicolumn{8}{|c|}{ Not responders } \\
\hline & 4 & 15 & $3+5$ & $\mathrm{~N}+$ & gonapeptyl + casodex RT & 52 & PSA/PSMA \\
\hline & 6 & 7,05 & $5+3$ & pT3b M+ & eligard + RT & 15 & PSA \\
\hline & 7 & 128 & $4+5$ & $\mathrm{~N}+\mathrm{M}+$ & eligard + casodex + taxotere & 68,5 & PSA/PSMA \\
\hline \multicolumn{8}{|c|}{$\begin{array}{l}\text { Responder patient } \\
\text { became not-responder }\end{array}$} \\
\hline & $2 *$ & 12 & $5+4$ & $\mathrm{~N}+\mathrm{M}+$ & eligard + casodex + RT + CHT & 6,81 & POS* \\
\hline
\end{tabular}

has confirmed the disease status; under strict radiological follow up, at distance metastases became evident three months later the molecular test resulted positive.

Patient n. 3: The test confirmed the efficacy of enlarged radiotherapy;

Patient n. 4: after lymphadenectomy, positive obturator lymph nodes were found at the extemporary frozen histological section: therefore radical prostatectomy was not performed but enlarged radiotherapy and total androgen blockade were administered. Since patient has become refractory to total androgen blockade, an "anti-androgen withdrawal" was started;

Patient n. 5: AdnaTest confirmed the efficacy of adjuvant postoperative RT;

Patient n. 6: he is currently under chemotherapy treatment because of the resistance also to second-line hormonal therapy

Patient n. 7: he is no longer responsive to chemotherapy since he showing constant disease progression;

Patient n. 12: despite a PSA increase was registered, disease progression is not yet evident. Since AdnaTest negative, a strict monitoring of PSA and CTCs overtime has been planned.

Patient n. 13: AdnaTest confirmed the good compliance associated to anti-androgen therapy suspension after radiotherapy;

Patient n. 14: AdnaTest supported the efficacy of adjuvant Radiotherapy after surgery;

Patient n. 16: (group II). AdnaTest, performed before surgery, confirmed the preoperative staging (definitive histological examination: pT2b R0 NO).

We underline that patients who responded to the therapies listed in Table 2 were always negative for Adna markers, while the non-responder resulted as positive for both serum PSA increases and prostate CTC markers.

\section{Discussion}

Several reports showed that circulating tumor cell counts correlate with prognosis in patients with advanced breast, prostate and colorectal cancers, treated by con- ventional and/or hormonal therapy, suggesting the use of CTCs in the clinical management of cancer patients $(18,27,28)$. Furthermore, for prognostic and predictive purposes, CTCs detection, when coupled to molecular characterisation of specific cell transcripts or biomarkers, could provide important clinical information in terms of monitoring of efficacy or resistance to targeted therapy: in this way, CTCs represent an accurate laboratory clinical tool for predicting patient's outcome also in prostate cancer and providing significant advantages in the view of personalised medicine (29). Over the past few years, different approaches for enrichment of CTCs in blood have been developed $(5,30-33)$, associated or not to molecular approaches and/or to immunological characterization, respectively (34-35).

The present preliminary study was aimed to assess the usefulness of AdnaTest Prostate Cancer for detection of CTCs in two groups of PCa patients (with low and high risk) as compared to non PCa individuals, in order to establish if the molecular result given by this assay could be helpful for the clinical and drug management of PCa patients. This test is, in fact, able to detect some prostate or cancer-specific markers such as PSA, PSMA and EGFR $(23,24)$.

In our study we found that AdnaTest: a) identified patients with disease progression or with biochemical relapse; b) confirmed the clinical staging of patient n.1, who showed biologically aggressive disease (GS 10) and biochemical tumor progression in spite of total androgen blockade or combined androgen blockade; c) likewise, in patient n. 2 , molecular test agreed firstly with the efficacy of chemotherapy, due to the blood negativity for AdnaTest during the first line of CHT, while only five months later AdnaTest resulted as positive, anticipating the results of radiological examination (the latter indicating at distance metastases only after three months from the evidence of a positive test for (TCs); d) AdnaTest was an useful predictive indicator for patient n.12, who was no more responsive to chemical castration so that anti-androgen therapy was currently discontinued. Patients enrolled in the present study are now under stringent follow-up, since they 
Figure 1A-B.

Amplification results obtained on a group of PCa patients.

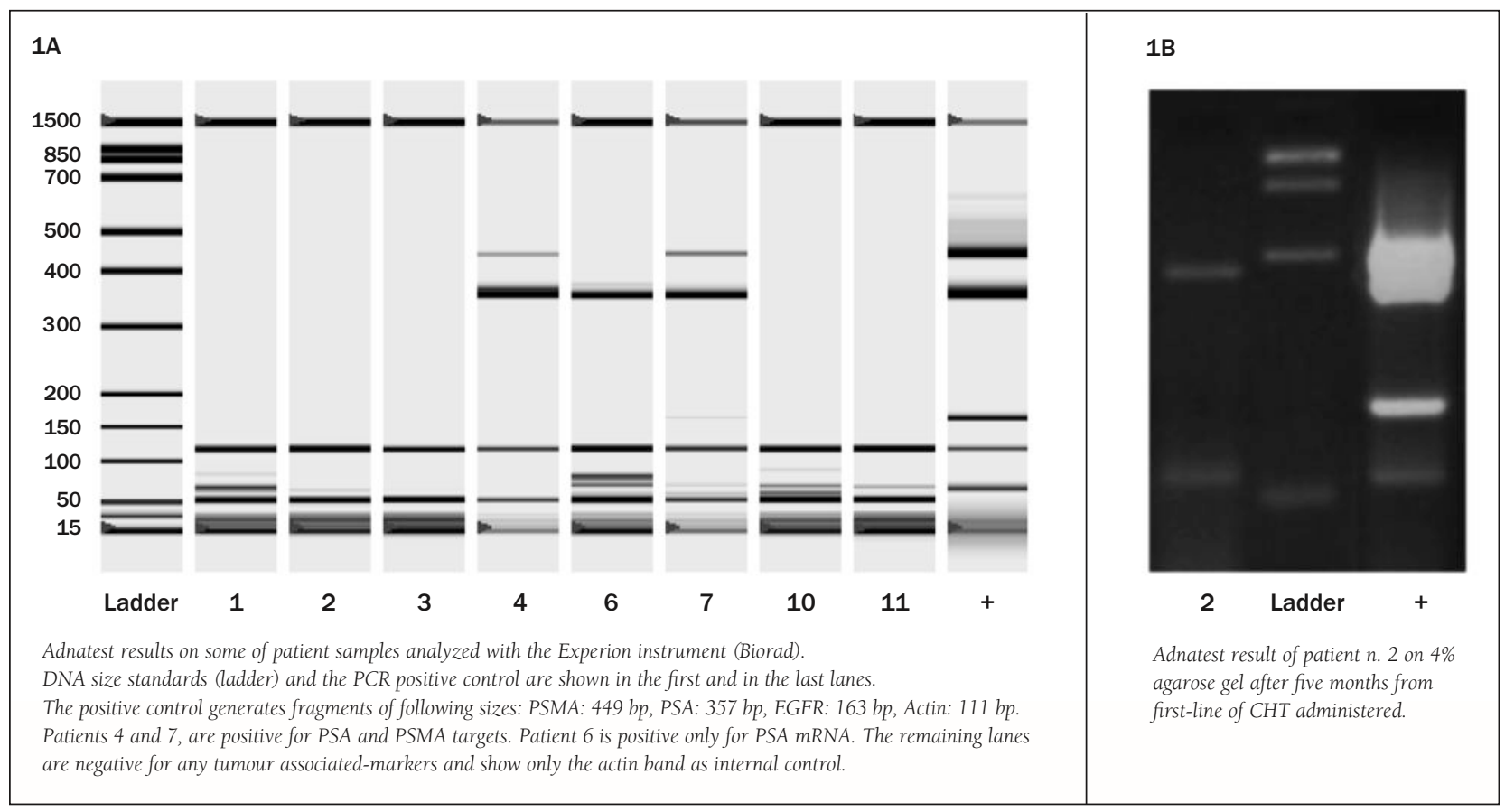

are followed by PSA assays overtime (every four months), and CT scan every six months, in order to plan other CTC tests for monitoring of disease progression. Regarding the molecular typing performed after enrichment, the use of multiplex AdnaGen PCR may improve the detection of at least one marker potentially associated to circulating prostate tumor cells and possibly associated to poor prognosis. Although mRNA-PSA is not unanimously considered as a better independent prognostic factor as compared to serum PSA detection (36), a limit of this test is that tumor-associated proteins, like PSA, are also expressed in normal cells. Nevertheless, in our test, when the suggested cut-off of $10 \mathrm{ng} / \mathrm{ml}$ was used, no false-positive results, particularly in BPH patients, were found. Furthermore, several studies suggested the use of PSMA, alone or combined to PSA-mRNA, to increase assay specificity in PCa patients (36), PSMA being over-expressed in advanced or in castration resistant PCa (37) and preferentially expressed in anaplastic cells, hormone-refractory cells and bone metastases (38).

\section{Conclusions}

In our opinion, molecular analysis of CTCs through PSMA mRNA detection could provide important information for clinicians in terms of prediction of disease recurrence, before imaging findings. This finding demonstrates the potential role of CTCs in the follow up of the Prostatic Cancer; it can be obtained in a routine practice setting, by a simple blood sampling.

We underline that although the present study did not compare the efficiency of AdnaTest with that of other platforms (in particular the CellSearch System), we can assume that our test may be considered as reliable: in fact, in this regard, Andreopoulou E et al have recently reported the concordance between these two methods (40). Finally, our results should be confirmed on larger cohorts of patients also considering that this type of test should be performed in laboratories with high expertise personnel in strict relationships with clinical departments.

\section{References}

1. Heidenreich A, Bolla M, Joniau S, et al. Guidelines on Prostate Cancer Eur Urol. 2011; 59:61-71 and 59:572-83.

2. Taplin ME, Bubley GJ, Shuster TD, et al. Mutation of the androgen-receptor gene in metastatic androgen-independent prostate cancer. N Engl J Med. 1995; 332:1393-8.

3. Chi KN, Bjartell A, Dearnaley D, et al. Castration-resistant prostate cancer: from new pathophysiology to new treatment targets. Eur Urol. 2009, 56:594-605.

4. Kruck S, Gakis G, Stenzl A. Circulating and disseminated tumor cells in the management of advanced prostate cancer. Adv Urol. 2012;135281. Epub 2011 Aug 21.

5. Paterlini-Brechot P, Benali NL. Circulating tumor cells (CTC) detection: clinical impact and future directions. Cancer Lett. 2007; 253:180-204.

6. Pantel K, Brakenhoff RH, Brandt B. Detection, clinical relevance and specific biological properties of disseminating tumour cells. Nat Rev Cancer. 2008; 8:329-40.

7. Allard WJ, Matera J, Miller MC, et al. Tumor cells circulate in the peripheral blood of all major carcinomas but not in healthy subjects or patients with non-malignant diseases. Clin Cancer Res. 2004; 10:6897-904

8. Jost M, Day JR, Slaughter R, et al. Molecular assays for the detection of prostate tumor derived nucleic acids in peripheral blood. Mol Cancer. 2010; 2:174. 
9. Moreno JG, Croce CM, Fischer R, et al. Detection of hematogenous micrometastasis in patients with prostate cancer. Cancer Res. 1992; 52:6110-12.

10. Katz AE, Olsson CA, Raffo AJ, et al. Molecular staging of prostate cancer with the use of an enhanced reverse transcriptasePCR assay. Urology. 1994; 43:765-75.

11. Sokoloff MH, Tso CL, Kaboo R, et al. Quantitative polymerase chain reaction does not improve preoperative prostate cancer staging: a clinicopathological molecular analysis of 121 patients. J Urol 1996; 156:1560-66.

12. Thiounn N, Saporta F, Flam TA, et al. Positive prostate-specific antigen circulating cells detected by reverse transcriptase-polymerase chain reaction does not imply the presence of prostatic micrometastases. Urology. 1997; 50:245-50.

13. Smalla AC, Gonga Y, Oh WK, et al. The Emerging Role of Circulating Tumor Cell Detection in Genitourinary Cancer. J Urol. 2012; 188:21-6.

14. Nagrath S, Sequist LV, Maheswaran S, et al. Isolation of rare circulating tumour cells in cancer patients by microchip technology. Nature. 2007; 450:1235-9.

15. Ellis WJ, Pfitzenmaier J, Colli J, et al. Detection and isolation of prostate cancer cells from peripheral blood and bone marrow. Urology. 2003; 61:277-81.

16. Moreno JG, Miller MC, Gross S, et al. Circulating tumor cells predict survival in patients with metastatic prostate cancer. Urology. 2005; 65:713-18.

17. Danila DC, Heller G, Gignac GA, et al. Circulating tumor cell number and prognosis in progressive castration-resistant prostate cancer. Clin Cancer Res. 2007; 13:7053-58.

18. de Bono JS, Scher HI, Montgomery RB, et al. Circulating tumor cells predict survival benefit from treatment in metastatic castration resistant prostate cancer. Clin Cancer Res. 2008; 14:6302-09.

19. Scher HI, Jia X, de Bono JS, et al. Circulating tumour cells as prognostic markers in progressive, castration-resistant prostate cancer: a reanalysis of IMMC38 trial data. Lancet Oncol. 2009; 10:233-9.

20. Stott SL, Lee RJ, Nagrath S, et al. Isolation and characterization of circulating tumor cells from patients with localized and metastatic prostate cancer. Sci Transl Med. 2010; 2:25ra23.

21. Rosenberg R, Gertler R, Friederichs J, et al. Comparison of two density gradient centrifugation systems for the enrichment of disseminated tumor cells in blood. Cytometry. 2002;49:150-58.

22. Todenhöfer T, Hennenlotter J, Feyerabend S, et al. Preliminary experience on the use of the Adnatest ${ }^{\circledR}$ system for detection of circulating tumor cells in prostate cancer patients. Anticancer Res. 2012; 32:3507-13.

23. Reynolds MA. Molecular alterations in prostate cancer. Cancer Lett. 2008; 271:13-24.

24. Bickers B, Aukim-Hastie C. New molecular biomarkers for the prognosis and management of prostate cancer-the post PSA era. Anticancer Res. 2009; 29:3289-98.

25. D'Amico AV, Whittington R, Broderick GA. Biochemical outcome after radical prostatectomy, external beam radiation therapy or interstitial radiation therapy for clinically localized prostate cancer. Jama. 1998; 280:969-74.

26. Benoy IH, Elst H, Van Dam P, et al. Detection of circulating tumour cells in blood by quantitative real-time RT-PCR: effect of pre-analytical time. Clin Chem Lab Med. 2006; 44:1082-7.

27. Cohen SJ, Punt C J, Iannotti N, et al. Relationship of circulating tumor cells to tumor response, progression-free survival, and overall survival in patients with metastatic colorectal cancer. J Clin Oncol. 2008; 26:3213-21.

28. Torino F, Bonmassar E, Bonmassar L, et al. Circulating tumor cells in colorectal cancer patients. Treat Rev. 2013 Jan 30. doi:pii: S0305-7372(13)00002-9. 10.1016/j.ctrv.2012.12.007.

29. Farace F, Massard C, Vimond N, et al. A direct comparison of CellSearch and ISET for circulating tumour-cell detection in patients with metastatic carcinomas. British Journal of Cancer. 2011; 105:847-53.

30. Alix-Panabieres C, Riethdorf S, Pantel K. Circulating tumor cells and bone marrow micrometastasis Clin. Cancer Res 2008; 14:5013-21.

31. Allan AL, Vantyghem SA, Tuck AB, et al. Detection and quantification of circulating tumor cells in mouse models of human breast cancer using immunomagnetic enrichment and multiparameter flow cytometry. Cytometry. 2005; 65:4-14.

32. Wiedswang $G$, Borgen E, Schirmer $C$, et al. Comparison of the clinical significance of occult tumor cells in blood and bone marrow in breast cancer. Int J Cancer. 2006; 118:2013-19.

33. Wong NS, Kahn HJ, Zhang L, et al. Prognostic significance of circulating tumour cells enumerated after filtration enrichment in early and metastatic breast cancer patients. Breast Cancer Res Treat. 2006; 99:63-9.

34. Stathopoulou A, Vlachonikolis I, Mavroudis D, et al. Molecular detection of cytokeratin-19-positive cells in the peripheral blood of patients with operable breast cancer: evaluation of their prognostic significance. J Clin Oncol. 2002; 20:3404-12.

35. Benoy IH, Elst H, Van der Auwera I, et al. Real-time RT-PCR correlates with immunocytochemistry for the detection of disseminated epithelial cells in bone marrow aspirates of patients with breast cancer. Br J Cancer. 2004; 91:1813-20.

36. Doyen J, Alix-Panabièresd C, Hofmanb P, et al. Circulating tumor cells in prostate cancer: A potential surrogate marker of survival. Crit Rev Oncol Hematol. 2012; 81:241-56.

37. Perner S, Hofer KR, Shah RB, et al. Prostate-specific membrane antigen expression as a predictor of prostate cancer progression. Hum Pathol. 2007; 38:696-701.

38. Israeli RS, Powell CT, Corr JG, et al. Expression of the prostatespecific membrane antigen. Cancer Res. 1994; 54:1807-11.

39. Sobin LH, Gospodariwicz M, Wittekind C. TNM classification of malignant tumors. UICC International Union Against Cancer. $7^{\text {th }}$ edn. Wiley-Blackwell. 2009; pp. 243-248.

40. Andreopoulou E, Yang LY, Rangel KM, et al. Comparison of assay methods for detection of circulating tumor cells in metastatic breast cancer: AdnaGen AdnaTest BreastCancer Select/Detect ${ }^{\mathrm{TM}}$ versus Veridex CellSearch ${ }^{\mathrm{TM}}$ system. Int J Cancer. 2012; 130:1590-7.

\section{Correspondence}

Giuseppe Albino, MD (Corresponding Author)

Urology Unit - Ospedale di Andria - ASL BAT

Ospedale "L. Bonomo", Andria, Italy

peppealbino@hotmail.com

Francesca Vendittelli, MD

Carmela Paolillo, MD

Cecilia Zuppi, MD

Ettore Capoluongo, MD

Laboratory of Molecular Diagnostics,

Institute of Biochemistry and Clinical Biochemistry,

Catholic University, Largo A. Gemelli 8 - 00168 Rome, Italy 